\title{
A METHOD OF FORESTRY DATA COLLECTION BASED ON ANDROID SYSTEM AND RTK
}

\author{
Y. Zeng ${ }^{1,2}$, G.W. Lan ${ }^{1,2}$, Z.G. Pan ${ }^{1,2}$, Y.L. Du ${ }^{1,2}$, H.L. Shi ${ }^{1,2}$ \\ ${ }^{1}$ Guangxi Key Laboratory of Spatial Information and Geomatics, Guilin University of Technology, Jiangan Road, Guilin, China - \\ (375308707, 23955461, 2277647553, 291296038, 1434546262)@qq.com \\ ${ }^{2}$ College of Geomatics and Geoinformation, Guilin University of Technology, Jiangan Road, Guilin, China
}

KEY WORDS: Android Mobile Terminal; RTK; Forestry Data Collection; High Precision Coordinate; Bluetooth

\begin{abstract}
:
In our country, forestry resource is one of the most important resources, which are of great significance to national economic and sustainable development. The operation mode of only using a mobile terminal to acquire data cannot satisfy the requirements of forestry data collection. A common mobile terminal is widely used to collect data with the advantage of convenient and rapid in field, but the error range of its GNSS chip positioning is 5 to 10 meters. Both positioning accuracy and the accuracy of sublot area measurement cannot meet the requirement in forestry data acquisition. Although RTK has the advantage of high- precision positioning, its handhold terminal is specially used for mobile single point positioning, and it cannot be suitable for forestry special data collection, storage and visualization. Therefore, this paper presents the idea of using bluetooth communication technology to wirelessly connect RTK with Android mobile terminal, the high precision coordinate is obtained and used for storage and display on the screen in real-time. Combining ArcGIS for Android development kit with SpatiaLite database, this paper develops a set of high-precision and intelligent forestry data acquisition system based on Android platform to realize real-time data collection and sublot editing. Compare with conventional forestry data collection method, the method combines the mobile terminal of Android system with RTK can improve work efficiency, which also provides a more efficient technical means for forestry data collection.
\end{abstract}

\section{INTRODUCTION}

Forest resource investigation means that collectors complete data collection, storage and management in the field, so as to help facilitate planning and design. In China, most of the forest resources located in remote areas with complex terrain and the number of species is large. The traditional methods of forest resource investigation require a large amount of human and material resources, data acquisition equipment is not abundant function and unfriendly operability, and many auxiliary tools have to be used for data entry, which leads to restricting the efficiency of field investigation (Zhou X H et al., 2018). In the early years, collectors use PDA with mobile GIS function to collect forestry data. the research and development of a complete data acquisition system provides a more convenient, more flexible, more efficient and faster way for the field data acquisition of forest resources. For example, a data acquisition system of PDA, developed by Xu Dengping, Tang Xiaoming, etc., it has some simple functions of data input and management, which is required to import the data into the computer for further processing (Xu D P et al., 2005). Zeng Songwei et al., from Zhejiang Agriculture and Forestry University, developed a system of forest resource investigation based on existing PDA. But the system has high redundancy and consumes large system resources (Zeng S W et al., 2009). As a mobile data acquisition equipment for forest resource inventory, PDA provides great convenience for the field staff, but its GNSS chip cannot meet the precision requirements for the point measurement, line measurement, area measurement and positioning in forest. Most field acquisition systems are only suitable for special surveys, they are poor integration and a low degree of automation. In recent years, with the continuous development of science and technology, the new technical operability of forestry data collection, investigation and planning has been continuously improved, the combination of GPS and GIS provides important technical means for precise forestry, which enriches the diverse methods of forest resource investigation and improves the overall level of forestry survey in an all-round and effective way (Zhu C R, 2018).

This paper presents a high-precision and intelligent method for data collection in forestry. Based on the requirements of data collection in forestry, it takes the advantage of the open-source features of android system and the convenience of data transmission as a mobile terminal. And stable bluetooth communication technology is used to realize the wireless connection with RTK and complete the high-precision coordinate acquisition. Combined with ArcGIS for Android development kit and SpatiaLite database, the system is carried out based on data information of geographical resource in forestry, which can realize real-time data collection and editing instead of carrying large quantities of drawings for comparative analysis, field marking and recording when going out for collection. The mobile terminal of android system is designed by this paper has integrating internal data processing and field data collection, which simplifies procedure of data collection and reduces the workload of data processing. It provides high precision and intelligent information services for data collection in forestry and better realize the construction of forestry data informatization. 


\section{FORESTRY DATA ANALYSIS}

\subsection{Data Requirement Analysis}

The work of forestry resource survey is to collect target area data by using android mobile terminal. Different types of data have significant differences in content, including structured data, semi-structured data and unstructured data. Which can be roughly divided into spatial data and attribute data (Chen $\mathrm{G}$ et al, 2012).

Spatial data, as an important symbol of geographic resource, it has become the core of data collection with characteristics of spatial diversity ( $\mathrm{Yu} \mathrm{Z} \mathrm{H,} \mathrm{2017).} \mathrm{Spatial} \mathrm{data} \mathrm{is} \mathrm{divided} \mathrm{into}$ non-editable map and editable vector data. Varies kind of forestry resource data are loaded into investigation system with the form of layer. Layers contains three types: point, line and polygon. The forestry resource data is mainly collected with the type of polygon. Android mobile terminal loads online map or offline image map processed by the desktop as base map, and then the editable vector data layer of the previous survey is superimposed on the base map, Collectors acquire vector data through field survey.

Attribute data is a description of multiple feature information for things, it is mainly in the form of a chart by text and tables. Different business forms have different content and data types. Attribute data editing is to revise the attribute table associated with spatial data. Collectors determine the data content to be collected according to the purpose of collection, and then establish corresponding form. Each data item to be collected serves as a field of the form. Finally, set the data type of field and the range of field values. The factor of sublot survey mainly include land type, soil, forest species, dominant species, forest age, area, etc. (2010). According to the requirements of forestry data collection, the attribute table correlated with spatial data is also set as the form of geographical location correlation, Which requires the attribute data to contain coordinate information and establish one to one correlation with sublot through Id. Partial data of the attribute form associated with spatial data are shown in table 1. Each sublot is identified by numerical sorting and set primary key constraints to make the data unique. Collectors selectively fills in the form data according to the actual situation of sublot. In addition, camera shooting is added to the collection of attribute data as a supplementary explanation, and the text information of attribute data is expressed in a more intuitive and rich way. The function of adding watermark time to the system photo is associated with time collected in sublot property table.

\begin{tabular}{|c|c|c|c|}
\hline Field name & Type & $\begin{array}{l}\text { Require } \\
\mathrm{d}\end{array}$ & Constraint \\
\hline Sublot Number & Number & Yes & Primary key \\
\hline Land Type & Number & Yes & \\
\hline Soil & Number & Yes & \\
\hline Forest Species & Number & No & \\
\hline $\begin{array}{c}\text { Dominant } \\
\text { Species }\end{array}$ & Number & No & \\
\hline Age Class & Number & Yes & \\
\hline Area & Number & Yes & \\
\hline Coordinate & Number & Yes & \\
\hline $\begin{array}{l}\text { Acquisition } \\
\text { Time }\end{array}$ & Number & Yes & \\
\hline & $\ldots$ & .. & \\
\hline
\end{tabular}

Table 1Partial data of sublot survey form The Coordinate filed in table 1 are obtained by RTK measurement technology and the area measurement is realized with high-precision coordinate points. RTK is carrier phase real-time differential technology, it can provide real-time three-dimensional positioning results of the station in the specified coordinate system and achieve centimeter-level accuracy (Wu S C, 2008). RTK is mainly composed of three parts: a reference station, a mobile station and an RTK handhold terminal. RTK handhold terminal has bluetooth module in the current market, it can collect coordinate data in any coordinate system. After the data is collected, it can be exported in TXT text file, KML file, CAD file and other formats. Android system provides a bluetooth API interface (Hou X Q, 2010). This paper develops bluetooth function and builds virtual serial port communication between android mobile terminal and RTK handhold terminal. So high-precision coordinate within the RTK are transmitted into Android mobile terminal and stored under the specified path, so that the system is taken to the real-time and effective coordinate information, and display coordinate information real-time on the map layer. Collectors draw coordinate points to realize sublot collection and area measurement.

From the above analysis, android mobile terminal should have functions of data editing, data input, data storage, and it can effectively process and manage data in real time. This article implements an android system based on ESRI's ArcGIS for Android development package which provides the interface of ArcGIS graphics processing and expression for developers, and it realizes spatial data editing, attribute data editing and entry functions mobile terminal. Spatialite space database as the data storage solution, not only solves the problem such as vector data storage and display, but also implements the unification of the spatial data and attribute data management, and ensure the integrity of the data.

\subsection{Data Collection Flow}

Filed collectors import the data of image map, sublot map and attribute table into the android mobile terminal. These data are made by indoor work. Collectors should make sure android mobile terminal, RTK and other auxiliary tools have good performance and plenty of electricity. Before arriving at a destination, collectors can use the positioning and navigation functions of the online map of android mobile terminal as direction guidance, and use the image map to compare with field to reach collection area faster and effectively, which can reduce the uncertainty in the data collection process (Tang $\mathrm{K} \mathrm{Y}$ et al, 2009). After arriving at the destination, collectors open the layer of previous survey sublot, and combine with the comparison of field landform. If the boundary of sublot does not change then input the attribute data directly. Or if the boundary of sublot is inconsistent with reality, the measured high-precision coordinates are transmitted to the sublot layer. Coordinates will be used as reference points to complete graph editing by hand drawing. Then collectors fill in form with the measurement results and attribute data to complete the current survey of forest land. The collection process of sublot is shown in figure 1 . 


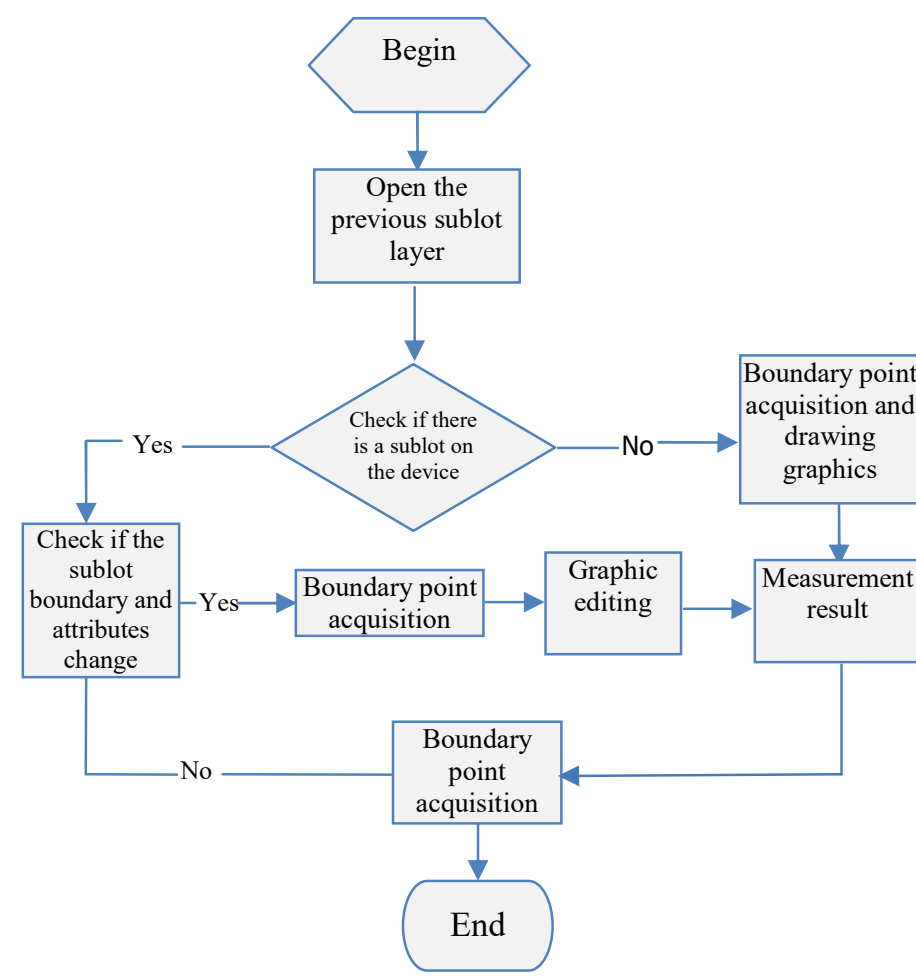

\subsection{System Design}

1. System Framework Design

The overall framework design of the system is shown in figure 2: The whole system is composed of RTK, android mobile terminal and PC terminal. The function of RTK is used to transmit the high-precision coordinates to the mobile terminal through the bluetooth interface, and assist the mobile terminal to collect the sublot boundary data. PC terminal is mainly responsible for data import and export with the mobile terminal and subsequent processing of the data. Android mobile terminal is the core part of the system. Forestry data collection system based on android system is mainly multi-functional software developed for collectors, which can be installed on android tablet computer or smart phone. The realization of application functions mainly adopts the development interfaces provided by android system. Developers can concentrate on software development with these interfaces without considering the interaction between software and the operating system.

Figure 1. Sublot acquisition process

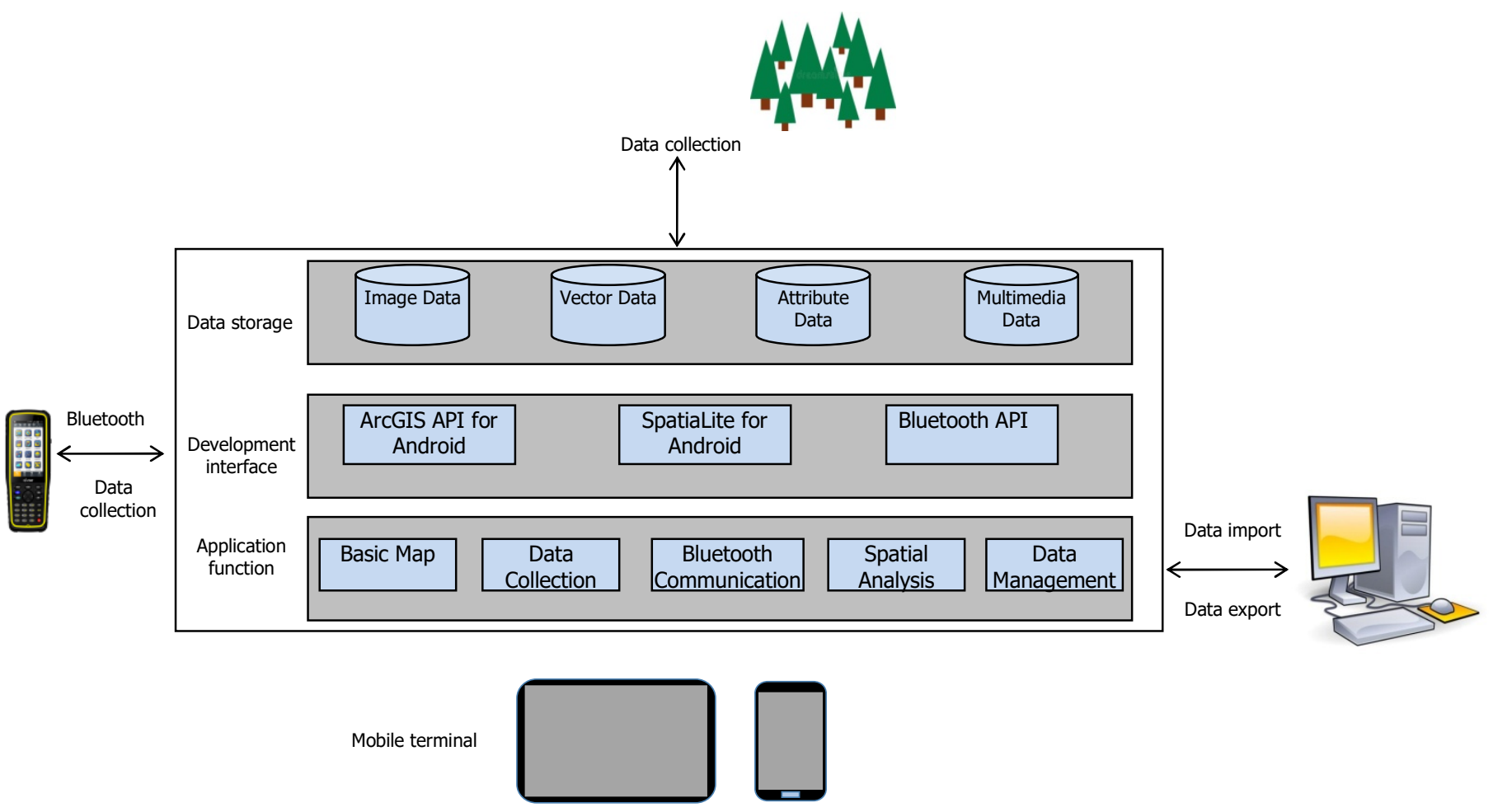

Figure 2. System function design 


\section{Function Design}

According to the business requirements of data collection, the main function design of this system is shown in figure 3

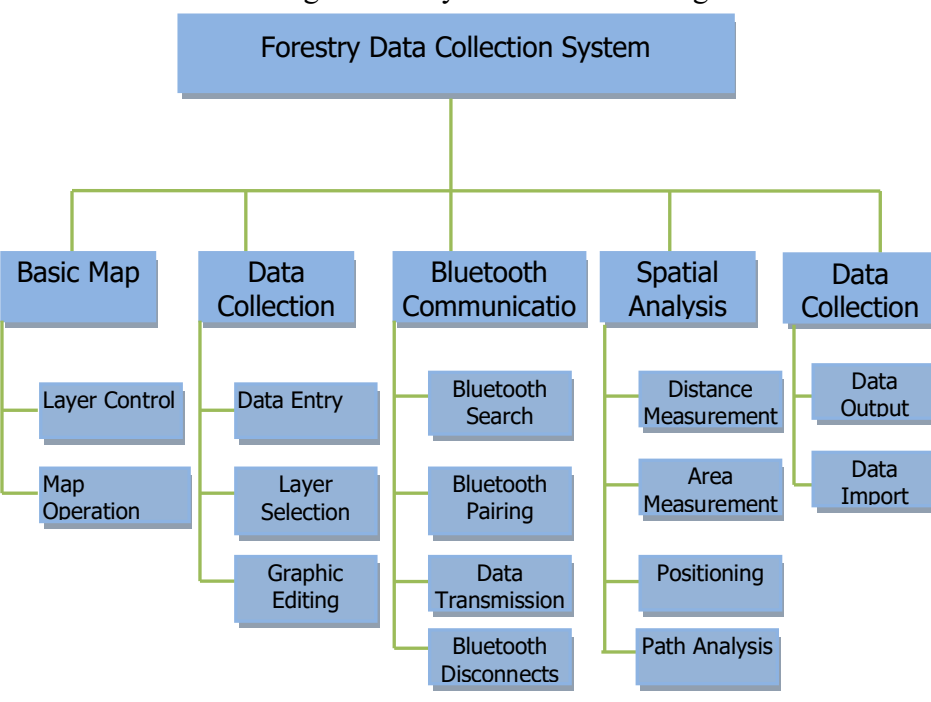

Figure 3. System function design

(1) Basic Map: Collectors can control display order of layer and select whether the layer to display or not. Map operations include zoom in, zoom out, pan and click.

(2) Data Collection: High-precision coordinates are real-time acquired by bluetooth. Multimedia data is recorded in the form of photographs. Collectors select editable vector layer to edit according to the target area. The editing of the graphic includes drawing, node editing, deleting. The editing of attribute data is to modify the attribute information table associated with spatial data

(3) Bluetooth Communication: Bluetooth module includes search, pairing, and data transmission, and disconnects after the data transmission are completed.

(4) Basic Tool: Spatial analysis provides line measurement and area measurement, and location and path analysis are used when the network is good.

(5) Data Management: It mainly completes the exchange function of system data information with other equipment, and realizes the synchronization of field data collection and internal industry work processing.

\subsection{Function Realization and Application}

Considering the network situation of field work, offline image data and vector data are processed by PC terminal. Android mobile terminal loads these offline data for editing, so as to ensure the field data can realize to butt with the office work. Hardware device consists of the following: Huawei glory 9 Youth Edition, operating system for Android 9, Zhonghaida V90 as RTK device.

The area of system application is a forest area in Guangxi Province, with a total area of 2,930 hectares and forest coverage of $92.5 \%$. In the forest, mountains are high, forests are densely covered, and animal and plant species are rich. Most of the native vegetation is the southern subtropical evergreen broad-leaved forest. It is highly representative from the perspective of forest resources distribution.

Due to the limited chapters, this paper mainly introduces the new method function of the system. The sublot in the system are numbered to identify different plots in forest. The data collection of sulobts are divided into drawing the non-existent map patches in the previous period and editing the existing map patches boundary, and then measure the distance and area of map patches.At last, the attribute data is entered after the measurement completed. Sublot data collect is shown in Figure 5.High-precision coordinates ared aquired by RTK bluetooth module, and used them to draw map patch, and then be highlighted in the mobile terminal. Map patches editing based on the high precision coordinates as the reference point,which is realized by dragging the node of existing map patches. In addition,the system has functions of snapping point and line when drawing map patches or editing map patches. After the map patches collection is finished, picture data can be collected by camera function. All attribute datas can be entered by clicking the ALL button on the attribute editing module. The attribute data entry table is shown in Figure 6.
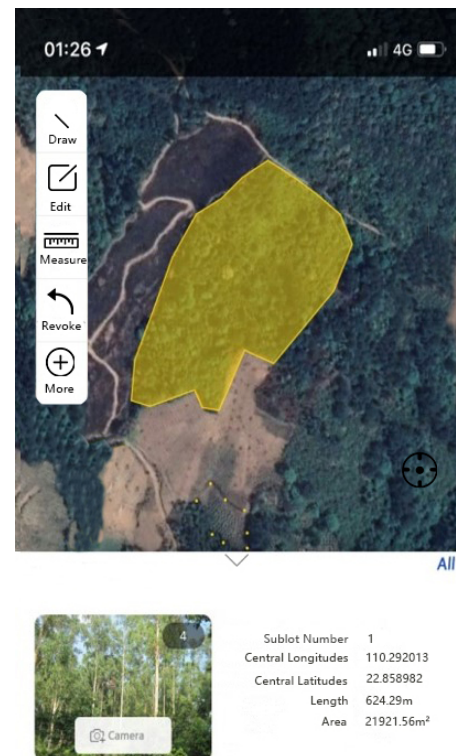

Figure5.Sublot data collect

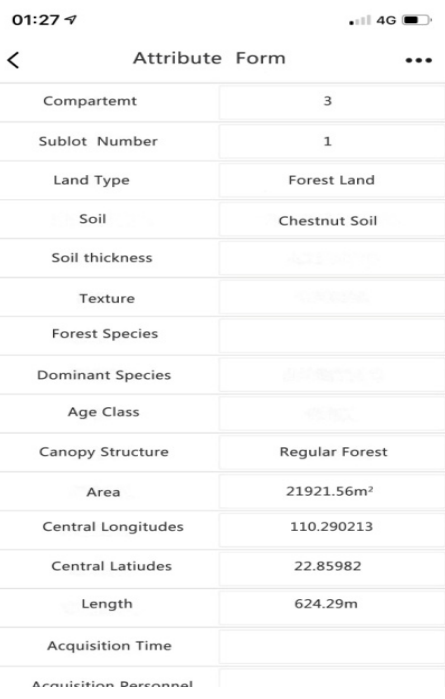

Figure 6.Attribute data entry 


\section{CONCLUSION}

First, this paper describes the current situation and development trend of mobile equipment for data collection of the wild forest resource. And then the key technologies required for the data acquisition of the forestry are studied. The design and development of the system aim to improve the accuracy and efficiency of the data acquisition, and it also adapt to the storage requirements of different data. Data of collection can be butted jointing data processing of interior work to realize the circulation of the internal and external data. RTK works with the android mobile terminal for forestry data collection has a great significance, which stores and manages data collected in the field uniformly, which reduces workflow, shortens work cycle and improves efficiency and economic benefits.

\section{ACKNOWLEDGEMENTS}

The research work is supported by National Natural Science Foundation of China under Grant No.41861050.

\section{REFERENCES}

Zhou, X, H., Xu, W, W.,2018. Comparative Analysis on the Field Collecting System and Traditional Class II Survey of the Forest Investigation Survey. Anhui Forestry Science and Technology.44(05):35-37.

Xu, D, P., Tang X, M., Wang, J, Z., Hu, H, S., 2005. Study on the PDA -based Forest Resources Data Collection System. Forest Resources Management. (01):58-62.

Zeng, S, W., Li, G, H., Hu, H, G., Tang, J, F., 2009. Design and implementation of personal digital assistant (PDA) based information collection system for forest resources. Forest Resources Management. 26(01):111-115.

Zhu, C, R., 2018. Discussion on the Application of GIS and GPS Technology in Forestry Investigation. Technology Innovation and Application. (6):140-141.

Chen, G., Liu, P, J., Li, Z, Q., Tang, X, M., 2012. Research of general data input system for forestry resource supervision. Journal of Beijing Forestry University. 34(06):92-97.

Yu, Z, H., 2017. Analysis of forest resource data characteristics. Inner Mongolia Forestry Investigation and Design. 40(03):83-84.

2010. Technical Regulations for Forest resource Planning and Design Survey.

Wu, S, C., 2008. Application Analysis of RTK Technology in Cadastral Survey. Science and Technology Information: Academic Research. (24):286+292

Li, L., Wang, Q., Wang, H, Q.,2010. GIS Data Management Based on Spatialite Database. Geomatics World. 8(04):71-75.

Hou, X, Q., Li, J., 2012. Research based on Android Bluetooth communication. Electronics World. (11):84-85.

Tang, K, Y., Tang, D, S., 2009.Research on the Uncertainty of Forest Resource Data. Sichuan Forestry Exploration and Design. (04):6-10. 